\title{
Stability of an Ultrarelativistic Blast Wave in an External Medium with a Steep Power\#Law Density Profile
}

\section{Citation}

Wang, Xiaohu, Abraham Loeb, and Eli Waxman. 2003. "Stability of an Ultrarelativistic Blast Wave in an External Medium with a Steep Power\#Law Density Profile." The Astrophysical Journal 594 (2): 924-35. https://doi.org/10.1086/377154.

\section{Permanent link}

http://nrs.harvard.edu/urn-3:HUL.InstRepos:41393353

\section{Terms of Use}

This article was downloaded from Harvard University's DASH repository, and is made available under the terms and conditions applicable to Other Posted Material, as set forth at http:// nrs.harvard.edu/urn-3:HUL.InstRepos:dash.current.terms-of-use\#LAA

\section{Share Your Story}

The Harvard community has made this article openly available. Please share how this access benefits you. Submit a story. 


\title{
STABILITY OF AN ULTRARELATIVISTIC BLAST WAVE IN AN EXTERNAL MEDIUM WITH A STEEP POWER-LAW DENSITY PROFILE
}

\author{
XiaOHU Wang AND ABraham LoEB ${ }^{1}$ \\ Department of Astronomy, Harvard University, 60 Garden Street, Cambridge, MA 02138; xwang@cfa.harvard.edu, aloeb@cfa.harvard.edu \\ AND \\ ELI WAXMAN \\ Department of Condensed Matter Physics, Weizmann Institute, Rehovot 76100, Israel; waxman@wicc.weizmann.ac.il \\ Received 2003 April 7; accepted 2003 May 24
}

\begin{abstract}
We examine the stability of self-similar solutions for an accelerating relativistic blast wave that is generated by a point explosion in an external medium with a steep radial density profile of a power-law index greater than 4.134. These accelerating solutions apply, for example, to the breakout of a gamma-ray burst outflow from the boundary of a massive star, as assumed in the popular collapsar model. We show that short wavelength perturbations may grow, but only by a modest factor $\lesssim 10$.
\end{abstract}

Subject headings: gamma rays: bursts — shock waves

\section{INTRODUCTION}

The self-similar solutions of relativistic blast waves are of much interest because of their recent applications to the study of gamma-ray bursts (GRBs). A sudden release of a large amount of energy within a small volume results in a strong explosion that drives a relativistic shock into the surrounding medium. At late times the blast wave approaches a self-similar phase, whereby its speed and the distribution of the pressure, density, and velocity of the gas behind the shock front do not depend on the length and timescales of the initial explosion, but only on the explosion energy and the properties of the unshocked external medium. The selfsimilar solutions describing this phase were first studied by Blandford \& McKee (1976, hereafter BMK). We list their central results, which are relevant to this paper, in $\S 2.1$. Note that in the BMK solution the total energy released in the explosion $E$ is the only relevant parameter.

The BMK solution is only valid for $k<4$, where $k$ is the power-law index of the radial density profile of the external medium, i.e., $\rho_{1} \propto r^{-k}$. When $k \geq 4$, the similarity variable defined by BMK is no longer appropriate. Even in the range $3 \leq k<4$, the validity of the BMK solution is not justified, because the mass contained behind the shock front diverges if the density profile of the shocked fluid is described by the BMK solution.

The self-similar solutions for steep density profiles with a power-law index $k \geq 4$ were derived recently by Best \& Sari (2000). ${ }^{2}$ The derivation of these solutions is similar to that in the nonrelativistic regime. The self-similar solutions to a nonrelativistic blast wave were discovered independently by Sedov (1946), Von Neumann (1947), and Taylor (1950). The so-called Sedov-Von Neumann-Taylor blast wave solutions exist only for $k<5$, but Waxman \& Shvarts (1993) showed that in the range $3 \leq k<5$ these solutions fail to describe the asymptotic flow because the energy diverges;

\footnotetext{
${ }^{1}$ Guggenheim Fellow. Currently on sabbatical leave at the Institute for Advanced Study, Princeton, NJ 08540.

2 The more extreme case of an exponential density profile has been discussed by Perna \& Vietri (2002).
}

instead they found second-type self-similar solutions for $3<k<5$ as well as for $k \geq 5$. The new class of nonrelativistic, self-similar solutions describes the flow in a limited spatial region $D(t) \leq r \leq R(t)$, where $R(t)$ is the shock radius and $D(t)$ coincides with a $C_{+}$characteristic so that the flow inside the region $r<D(t)$ does not affect the flow in the outer self-similar region. The self-similar solution has to cross the sonic line into the region where the $C_{+}$characteristic cannot catch up with the shock front. The solution describes a shock accelerating with a temporal dependence whose power-law index is uniquely determined by requiring that the self-similar solution cross the sonic line at a singular point. Note that in these second-type self-similar solutions the total energy released in the explosion $E$ is not the determining parameter. ${ }^{3}$ Although the energy in the self-similar part of the flow approaches a constant as time diverges, the fraction of the explosion energy $E$ carried by the self-similar component depends on the details of the initial conditions. Thus, contrary to the BMK case, dimensional arguments cannot be used to determine the power-law index of the temporal dependence. Instead, the singular point determines the temporal power-law index. In the ultrarelativistic regime, the second-type self-similar solutions for $k \geq 4$ can similarly be obtained by requiring that they cross the sonic line at a singular point. Best \& Sari (2000) found that these self-similar solutions exist for $k>5-(3 / 4)^{1 / 2}$ and describe accelerating shock waves. However, the properties of the flow in the self-similar region, such as the energy and mass contained in the region, were not discussed.

In this paper, we rederive the self-similar solutions of ultrarelativistic blast waves for $k>4$ using a different selfsimilar variable and discuss the properties of the flow in the self-similar regime. Our main goal is to study the stability of these self-similar solutions. The stability of the WaxmanShvarts self-similar solutions in the nonrelativistic regime was studied by Sari, Waxman, \& Shvarts (2000). They found that shocks accelerating at a rate larger than a critical

\footnotetext{
${ }^{3}$ For a comprehensive discussion on the second type of self-similar motion, see Zeldovich \& Raizer (2002).
} 
value and corresponding to solutions that diverge in finite time are unstable for small and intermediate wavenumbers. Shocks that accelerate at a rate smaller than the critical rate are stable for most wavenumbers. The acceleration rate can be quantified by the measure $\delta=R \ddot{R} / \dot{R}^{2}$, where the dots denote time derivatives and $R(t)$ is the radius of the shock front. This measure provides the fractional change of the velocity over a characteristic timescale for evolution $R / \dot{R}$. Solutions that diverge in finite time have $\delta>1$, while others have $\delta<1$. Thus, when shocks accelerate sufficiently fast they become unstable.

In the following sections we study the stability of the selfsimilar solutions of ultrarelativistic blast waves for steep density profiles with a power-law index $k>4$. The selfsimilar solutions are described in $\S 2$. We list the BMK solutions for $k<4$ in $\S 2.1$ and derive the self-similar solutions for $k>4$ in $\S 2.2$. In $\S 3$, we discuss the properties of the self-similar flow and calculate the energy and mass contained in the self-similar regime. In $\S \S 4$ and 5, we study the stability of the self-similar solutions. Finally, we summarize our main results in $\S 6$.

\section{SELF-SIMILAR SOLUTIONS}

\subsection{BMK Solutions for $k<4$}

For pedagogical reasons, we first briefly outline the derivation of the self-similar solutions of relativistic blast waves for $k<4$ by BMK. For a complete derivation, the reader is referred to the original paper (BMK).

Assuming an ultrarelativistic equation of state, $p=\frac{1}{3} e$, where $p$ and $e$ are the pressure and energy density, respectively, measured in the fluid frame, the equations describing a relativistic, spherically symmetric, perfect fluid can be written as

$$
\begin{gathered}
\frac{d}{d t}\left(p \gamma^{4}\right)=\gamma^{2} \frac{\partial p}{\partial t}, \\
\frac{d}{d t} \ln \left(p^{3} \gamma^{4}\right)=-\frac{4}{r^{2}} \frac{\partial}{\partial r}\left(r^{2} \beta\right), \\
\frac{\partial n^{\prime}}{\partial t}+\frac{1}{r^{2}} \frac{\partial}{\partial r}\left(r^{2} n^{\prime} \beta\right)=0,
\end{gathered}
$$

where $n^{\prime}$ is the density as measured in the laboratory frame, $\gamma$ and $\beta$ are the Lorentz factor and velocity of the fluid, respectively, and

$$
\frac{d}{d t} \equiv \frac{\partial}{\partial t}+\beta \frac{\partial}{\partial r}
$$

is the convective derivative. Throughout this paper we set the speed of light $c$ to unity. Assuming that the blast wave is ultrarelativistic so that the Lorentz factor of the shock front $\Gamma$ and the shocked fluid $\gamma$ are much larger than unity, we only search for solutions accurate to the lowest order in $\gamma^{-2}$ and $\Gamma^{-2}$.

The effective thickness of the blast wave is approximately $R / \Gamma^{2}$, where $R$ is the radius of the shock front. Thus, an appropriate choice of similarity variable is

$$
\xi=\left(1-\frac{r}{R}\right) \Gamma^{2} \geq 0 .
$$

Next we assume that the external medium has a scale-free, power-law density profile $\rho_{1} \propto r^{-k}$. Ignoring radiative losses, the total energy contained in the shocked fluid remains constant, and so the Lorentz factor of the shock front evolves adiabatically as a power law:

$$
\Gamma^{2} \propto t^{-m}, \quad m>-1 .
$$

Keeping only terms up to the order $O\left(\Gamma^{-2} t\right)$, the shock radius is then given by

$$
R=t\left[1-\frac{1}{2(m+1) \Gamma^{2}}\right]
$$

A more convenient similarity variable can be defined as

$$
\chi=1+2(m+1) \xi=\left[1+2(m+1) \Gamma^{2}\right]\left(1-\frac{r}{t}\right) .
$$

In terms of $\chi$, the pressure, velocity, and density in the shocked fluid can be written as, respectively,

$$
\begin{gathered}
p=\frac{2}{3} w_{1} \Gamma^{2} f(\chi), \\
\gamma^{2}=\frac{1}{2} \Gamma^{2} g(\chi), \\
n^{\prime}=2 n_{1} \Gamma^{2} h(\chi),
\end{gathered}
$$

where $\chi \geq 1$ and $w_{1}$ and $n_{1}$ are the enthalpy and number density, respectively, of the unshocked external medium. We assume that the unshocked external medium is cold, so that $w_{1}$ equals the energy density $\rho_{1}$. The jump conditions for a strong ultrarelativistic shock are satisfied by the boundary conditions

$$
f(1)=g(1)=h(1)=1
$$

For an adiabatic impulsive blast wave, equations (1)-(3) admit a simple analytical solution, first derived by BMK,

$$
\begin{gathered}
f=\chi^{-(17-4 k) /(12-3 k)}, \\
g=\chi^{-1}, \\
h=\chi^{-(7-2 k) /(4-k)},
\end{gathered}
$$

for

$$
m=3-k>-1
$$

\subsection{Self-similar Solutions for $k>4$}

In searching for self-similar solutions for $k>4$, we assume that the Lorentz factor of the shock front still obeys a power law, $\Gamma^{2} \propto t^{-m}$, with $m<-1$. Therefore, these selfsimilar solutions describe accelerating shock waves. When $m<-1$, the similarity variable $\chi$ defined in equation (8) (and used by Best $\&$ Sari 2000) could be negative. For convenience we will use $\xi$, defined in equation (5), instead as our similarity variable. If at an initial time $t_{0}$, the shock radius is $R_{0}$ and the Lorentz factor of the shock front is $\Gamma_{0}$, then at a later time $t$ to $O\left(\Gamma^{-2} t\right)$ the shock radius is given by

$$
R=R_{0}+t\left[1-\frac{1}{2(m+1) \Gamma^{2}}\right]-t_{0}\left[1-\frac{1}{2(m+1) \Gamma_{0}^{2}}\right] .
$$

We can rewrite this equation as

$$
R=t\left[1-\frac{1}{2(m+1) \Gamma^{2}}\right]+a,
$$


where $a$ is a constant dictated by the initial conditions. This equation for $R$ with $m<-1$ differs from equation (7) by a constant $a$. However, we can choose the initial time $t_{0}$ such that $a$ is equal to zero. This is appropriate for two reasons. First, the self-similar solutions are valid at much later times $t \gg t_{0}$; thus, the effect of the special choice of $t_{0}$ can be ignored. Second, what matters in the derivation of the selfsimilar solutions is the derivative of $R$ instead of $R$ itself. When $a=0$, the similarity variable becomes

$$
\xi=\left(1-\frac{r}{R}\right) \Gamma^{2}=\Gamma^{2}-\frac{r}{t}\left[\Gamma^{2}+\frac{1}{2(m+1)}\right] .
$$

Note that we have ignored higher order terms in $\Gamma^{-2}$ in the above expression.

Similarly to equations (9)-(11), we write the pressure, velocity, and density in the shocked fluid as, respectively,

$$
\begin{gathered}
p=\frac{2}{3} w_{1} \Gamma^{2} f(\xi), \\
\gamma^{2}=\frac{1}{2} \Gamma^{2} g(\xi), \\
n^{\prime}=2 n_{1} \Gamma^{2} h(\xi),
\end{gathered}
$$

where $\xi \geq 0$ and the boundary conditions,

$$
f(0)=g(0)=h(0)=1,
$$

correspond to the jump conditions for a strong ultrarelativistic shock.

We can now treat $\Gamma^{2}$ and $\xi$ as two new independent variables in place of $r$ and $t$ and get

$$
\begin{gathered}
t \frac{\partial}{\partial t}=-m \Gamma^{2} \frac{\partial}{\partial \Gamma^{2}}+\left[\Gamma^{2}-\frac{1}{2} \frac{m}{m+1}-(m+1) \xi\right] \frac{\partial}{\partial \xi}, \\
t \frac{\partial}{\partial r}=-\left[\Gamma^{2}+\frac{1}{2(m+1)}\right] \frac{\partial}{\partial \xi}, \\
t \frac{d}{d t}=-m \Gamma^{2} \frac{\partial}{\partial \Gamma^{2}}-\left[\frac{1}{2}+(m+1) \xi-\frac{1}{g}\right] \frac{\partial}{\partial \xi} .
\end{gathered}
$$

In deriving the above equations, we have assumed that the blast wave is ultrarelativistic so that $\Gamma \gg 1$ and $\gamma \gg 1$. Thus, we only keep terms of the lowest contribution order in $\Gamma^{-2}$ and $\gamma^{-2}$.

Substituting equations (24)-(26) into equations (1)-(3), we obtain the following differential equations for $f, g$, and $h$ :

$$
\begin{aligned}
2(3 m+k) g+[g & +2(m+1) g \xi+2] \frac{1}{f} \frac{d f}{d \xi} \\
& +2[g+2(m+1) g \xi-2] \frac{1}{g} \frac{d g}{d \xi}=0, \\
2(5 m+3 k-8) g & +3[g+2(m+1) g \xi-2] \frac{1}{f} \frac{d f}{d \xi} \\
& +2[g+2(m+1) g \xi+2] \frac{1}{g} \frac{d g}{d \xi}=0,
\end{aligned}
$$

$2(m+k-2) g+[g+2(m+1) g \xi-2] \frac{1}{h} \frac{d h}{d \xi}+2 \frac{1}{g} \frac{d g}{d \xi}=0$.

Using a new variable,

$$
y=[1+2(m+1) \xi] g,
$$

we rewrite equations (27)-(29) as follows:

$$
\begin{aligned}
& \frac{1}{g}\left(\frac{1}{f} \frac{d f}{d \xi}\right)=\frac{2[4(2 m+k-2)-(m+k-4) y]}{y^{2}-8 y+4} \\
& \frac{1}{g}\left(\frac{1}{g} \frac{d g}{d \xi}\right)=\frac{2[(7 m+3 k-4)-(m+2) y]}{y^{2}-8 y+4} \\
& \frac{1}{g}\left(\frac{1}{h} \frac{d h}{d \xi}\right)=-2\left[\left(y^{2}-8 y+4\right)(y-2)\right]^{-1} \\
& \times\left[(m+k-2) y^{2}-(10 m+8 k-12) y\right. \\
&+(18 m+10 k-16)] .
\end{aligned}
$$

One solution to the above equations is obtained for $y=1$ and $m=3-k$ and corresponds to the BMK solution. From the definition of $y, y=[1+2(m+1) \xi] g$, and the requirement that $g$ be positive, it follows that this solution is only valid for $m>-1$, i.e., $k<4$.

In our search for possible solutions with $k>4$, we start by analyzing equations (31)-(33). The right-hand sides of these equations diverge to infinity if $y^{2}-8 y+4=0$. This corresponds to two singular points, $y_{1}=4-2 \sqrt{3}=0.536$ and $y_{2}=4+2 \sqrt{3}=7.464$. In addition, equation (33) has another singular point at $y_{3}=2$. The solution to equation (31) can bypass the singular points $y_{1}$ and $y_{2}$ if the numerator on the right-hand side of the equation vanishes at $y_{1}$ or $y_{2}$. This gives

$$
m_{1,2}=\frac{8-4 k+(k-4) y_{1,2}}{8-y_{1,2}} .
$$

It is easy to prove that when $m=m_{1}$, equations (32) and (33) will also bypass the singular point $y_{1}$ (the numerator in the right-hand side of each equation is equal to zero at $y_{1}$ ). The same is true for $m=m_{2}$ and the singular point $y_{2}$. We will show below that when $m=m_{1}$ and $k$ is bigger than a critical value $k_{c}$, we have $y \leq 1$; thus, equations (31)-(33) are able to bypass the singular point $y_{1}$ and never reach $y_{2}$ and $y_{3}$. The critical value $k_{c}$ can be calculated by setting $m_{1}$ equal to $3-k$, the $m$-value corresponding to a BMK solution. For $y_{1}=4-2 \sqrt{3}$, we get

$$
m_{1}=12 \sqrt{3}-20+(3-2 \sqrt{3}) k .
$$

Thus, $m_{1}=3-k$ gives us

$$
k_{c}=5-\frac{\sqrt{3}}{2}=4.134 .
$$

The value of $m_{1}$ corresponding to $k_{c}$ is $m_{1 c}=$ $-2+\sqrt{3} / 2=-1.134$. Thus, when $k>k_{c}$, we have $m_{1}<m_{1 c}$.

We now prove that when $m=m_{1}$ and $k>k_{c}$, we always have $y \leq 1$. Using equation (32) and the definition of $y$ in equation (30), we obtain

$$
\begin{aligned}
\frac{d y}{d \xi} & =2(m+1) g(\xi)+\frac{y}{g(\xi)} \frac{d g}{d \xi} \\
& =-\frac{2 g(\xi)\left[y^{2}+(m-3 k+12) y-4(m+1)\right]}{y^{2}-8 y+4} .
\end{aligned}
$$

When $m=m_{1}$, the above equation can be rewritten as

$$
\frac{d y}{d \xi}=-\frac{2 g(y-b)}{y-y_{2}}
$$


where

$$
b=4-10 \sqrt{3}+2 \sqrt{3} k=1+2 \sqrt{3}\left(k-k_{c}\right) .
$$

When $k>k_{c}$, we have $b>1$. We also have $g>0$ and $y_{2}=4+2 \sqrt{3}>1$, and so the right-hand side of equation (38) is negative when $y \leq 1$. Since the boundary condition is $y(\xi=0)=1, y(\xi)$ must be a monotonically decreasing function of $\xi$ with $y(\xi) \leq 1$. The asymptotic behavior of $y(\xi)$ can be derived as follows. When $\xi$ is large, $y$ is negative and $|y|$ is large so that equation (38) can be approximated as

$$
\frac{d y}{d \xi} \simeq-2 g \simeq-\frac{1}{m_{1}+1} \frac{y}{\xi},
$$

where we have used the approximation $y \simeq 2\left(m_{1}+1\right) \xi g$ for large $\xi$. Equation (40) yields

$$
-y \propto \xi^{-1 /\left(m_{1}+1\right)} .
$$

Note that when $k>k_{c}$, we have $m_{1}<m_{1 c}<-1$. Thus, the exponent in the above power law is always positive.

It can be proved that when $k \leq k_{c}$, equations (31)-(33) cannot bypass all the singular points with either $m_{1}$ or $m_{2}$, and so the BMK solution is the only possible solution. When $k>k_{c}$, the equations cannot bypass all the singular points with $m_{2}$. But this by itself is not sufficient for justifying that $m_{1}$ is the only viable choice. What if the solutions cut off at some radius before reaching any singular points? We know that in order not to run into divergences of the energy or mass of the system, the solutions must be truncated at some radius $r_{+}$(or $\xi_{+}$in terms of the similarity variable), which should coincide with a $C_{+}$characteristic line. ${ }^{4}$ The $C_{+}$characteristic guarantees that the flow in the inner region $r<r_{+}$will not influence the flow in the selfsimilar region $r_{+} \leq r \leq R$. This $C_{+}$characteristic should not overtake the shock front in finite time; otherwise the selfsimilar region will eventually disappear. This argument has been applied in the nonrelativistic case (Waxman \& Shvarts 1993). We will prove below that in order to get to the regime where the $C_{+}$characteristics cannot catch the shock front, the solutions have to pass the singular point $y_{1}$, making $m_{1}$ the only viable choice.

First, let us derive the equation for a $C_{+}$characteristic. We use $v$ to denote the fluid velocity in the laboratory frame. The sound speed in the fluid frame is $u_{s}^{\prime}=1 / \sqrt{3}$. Thus, the sound speed in the laboratory frame $u_{s}$ is given by

$u_{s}=\frac{u_{s}^{\prime}+v}{1+v u_{s}^{\prime}}=1-\frac{\sqrt{3}-1}{\sqrt{3}+1} \frac{1}{2 \gamma^{2}}=1-\frac{\sqrt{3}-1}{\sqrt{3}+1} \frac{1}{\Gamma^{2} g(\xi)}$,

where we only keep the first-order term in $\gamma^{-2}$. Thus, a $C_{+}$ characteristic is described by

$$
\frac{d r_{+}}{d t}=1-\frac{\sqrt{3}-1}{\sqrt{3}+1} \frac{1}{\Gamma^{2} g\left(\xi_{+}\right)} .
$$

We can rewrite this equation in terms of the similarity variable $\xi_{+}$. Using the definition of $\xi$ in equation (5), and the relations $d R / d t=1-1 /\left(2 \Gamma^{2}\right)$ and $d \Gamma^{2} / d t=-m \Gamma^{2} / t$, we

\footnotetext{
${ }^{4}$ For a discussion on the nature of $C_{+}$and $C_{-}$characteristics in onedimensional relativistic hydrodynamics, see Johnson \& McKee (1971).
}

obtain

$$
\frac{d r}{d t}=1-\frac{1}{2 \Gamma^{2}}-\frac{(m+1)}{\Gamma^{2}} \xi-\frac{t}{\Gamma^{2}} \frac{d \xi}{d t},
$$

where we only keep the first-order term in $\Gamma^{-2}$. Substituting equation (44) into equation (43), we get

$$
t \frac{d \xi_{+}}{d t}=\frac{\sqrt{3}-1}{\sqrt{3}+1} \frac{1}{g\left(\xi_{+}\right)}-(m+1) \xi_{+}-\frac{1}{2},
$$

which describes the evolution of a $C_{+}$characteristic. We can further rewrite this equation as

$$
t \frac{d \xi_{+}}{d t}=-\frac{1}{2 g\left(\xi_{+}\right)}\left(y_{+}-y_{1}\right)
$$

where

$$
y_{+}=\left[1+2(m+1) \xi_{+}\right] g\left(\xi_{+}\right) .
$$

Equation (46) implies that when $y_{+}>y_{1}$, the right-hand side of the equation is negative, and so $\xi_{+}$will decrease with time and the $C_{+}$characteristic will approach the shock front. Only when $y_{+}<y_{1}$ will $\xi_{+}$increase with time, and the $C_{+}$characteristic will not overtake the shock front. Also notice that the self-similar solution has the boundary condition $y(\xi=0)=1>y_{1}$. We thus proved that in order to get to the regime where $C_{+}$characteristics cannot overtake the shock front, the self-similar solution must pass through the singular point $y_{1}$, and therefore $m_{1}$ is the only viable choice.

We can now attempt to obtain the self-similar solutions for equations (31)-(33). For $m=m_{1}$, these equations become

$$
\begin{gathered}
\frac{1}{g}\left(\frac{1}{f} \frac{d f}{d \xi}\right)=-\frac{2\left(m_{1}+k-4\right)}{y-y_{2}}, \\
\frac{1}{g}\left(\frac{1}{g} \frac{d g}{d \xi}\right)=-\frac{2\left(m_{1}+2\right)}{y-y_{2}}, \\
\frac{1}{g}\left(\frac{1}{h} \frac{d h}{d \xi}\right)=-\frac{2\left(m_{1}+k-2\right)(y-d)}{\left(y-y_{2}\right)(y-2)},
\end{gathered}
$$

where

$$
d=4+\sqrt{3}+\frac{3+2 \sqrt{3}}{k+\sqrt{3}-4} .
$$

Treating $y$ as the independent variable instead of $\xi$ and making use of equation (38), equations (48)-(50) can be rewritten as

$$
\begin{gathered}
\frac{1}{f} \frac{d f}{d y}=\frac{m_{1}+k-4}{y-b}, \\
\frac{1}{g} \frac{d g}{d y}=\frac{m_{1}+2}{y-b}, \\
\frac{1}{h} \frac{d h}{d y}=\frac{\left(m_{1}+k-2\right)(y-d)}{(y-2)(y-b)} .
\end{gathered}
$$

The boundary conditions are $f(y=1)=g(y=1)=$ $h(y=1)=1$. Equations (52) and (53) have the solutions

$$
f=\left(\frac{b-y}{b-1}\right)^{m_{1}+k-4},
$$




$$
g=\left(\frac{b-y}{b-1}\right)^{m_{1}+2} .
$$

A special case is obtained at $k=6\left(m_{1}=-2\right)$, for which $f(y)=g(y)=1$. When $b \neq 2[k \neq(15-\sqrt{3}) / 3]$, equation (54) has the solution

$$
h=\left[\left(\frac{1}{2-y}\right)^{d-2}\left(\frac{b-y}{b-1}\right)^{d-b}\right]^{\left(m_{1}+k-2\right) /(2-b)} .
$$

When $b=2[k=(15-\sqrt{3}) / 3]$, equation (54) has the solution

$$
\begin{aligned}
h= & (2-y)^{m_{1}+k-2} \\
& \times \exp \left[\left(m_{1}+k-2\right)(d-2)\left(\frac{1}{2-y}-1\right)\right] .
\end{aligned}
$$

In general, the functions $f(\xi), g(\xi)$, and $h(\xi)$ do not admit simple analytical forms. Their values can be derived numerically from equations (55)-(58). For example, $g(\xi)$ satisfies the implicit algebraic equation

$$
g(\xi)=\left\{\frac{b-\left[1+2\left(m_{1}+1\right) \xi\right] g(\xi)}{b-1}\right\}^{m_{1}+2} .
$$

But generally, we can derive the analytical forms for the asymptotic behaviors of $f(\xi), g(\xi)$, and $h(\xi)$ in the limit of large $\xi$. In this limit, $y$ is negative and $|y|$ is large, and so equation (56) yields

$$
g \simeq\left(\frac{-y}{b-1}\right)^{m_{1}+2} \simeq\left[\frac{-2\left(m_{1}+1\right) \xi g}{b-1}\right]^{m_{1}+2} .
$$

We can solve $g(\xi)$ from the above equation and get

$$
g(\xi) \simeq \alpha_{g} \xi^{-\left(m_{1}+2\right) /\left(m_{1}+1\right)},
$$

where

$$
\alpha_{g}=\left[\frac{-2\left(m_{1}+1\right)}{b-1}\right]^{-\left(m_{1}+2\right) /\left(m_{1}+1\right)} .
$$

Using equation (61), we obtain the asymptotic form of $y(\xi)$ for large $\xi$ :

$$
y(\xi) \simeq-\alpha_{y} \xi^{-1 /\left(m_{1}+1\right)}
$$

where

$$
\alpha_{y}=\left[\frac{-2\left(m_{1}+1\right)}{(b-1)^{m_{1}+2}}\right]^{-1 /\left(m_{1}+1\right)}
$$

Using equation (63), we can derive the asymptotic form of $f(\xi)$ for large $\xi$ from equation (55) and get

$$
f(\xi) \simeq \alpha_{f} \xi^{-\left(m_{1}+k-4\right) /\left(m_{1}+1\right)},
$$

where

$$
\alpha_{f}=\left[\frac{-2\left(m_{1}+1\right)}{b-1}\right]^{-\left(m_{1}+k-4\right) /\left(m_{1}+1\right)} .
$$

Similarly, the asymptotic form of $h(\xi)$ for large $\xi$ can be derived from equations (57) and (58). We obtain

$$
h(\xi) \simeq \alpha_{h} \xi^{-\left(m_{1}+k-2\right) /\left(m_{1}+1\right)},
$$

where

$$
\alpha_{h}=\left[\frac{-2\left(m_{1}+1\right)}{(b-1)^{1-\left(m_{1}+1\right)(d-2) /(2-b)}}\right]^{-\left(m_{1}+k-2\right) /\left(m_{1}+1\right)}
$$

if $b \neq 2$ and

$$
\begin{aligned}
\alpha_{h}= & {\left[\frac{-2\left(m_{1}+1\right)}{(b-1)^{m_{1}+2}}\right]^{-\left(m_{1}+k-2\right) /\left(m_{1}+1\right)} } \\
& \times \exp \left[-\left(m_{1}+k-2\right)(d-2)\right]
\end{aligned}
$$

if $b=2$.

Equation (46), which describes the evolution of the $C_{+}$ characteristic, can also be rewritten using the variable $y$. Using equation (38), we obtain

$$
t \frac{d y_{+}}{d t}=\frac{\left(y_{+}-y_{1}\right)\left(y_{+}-b\right)}{y_{+}-y_{2}} .
$$

We have proved earlier that when $k>k_{c}$, one finds $b>1$ and $y \leq 1$. Thus, the sign of the right-hand side of the above equation is decided by the term $\left(y_{+}-y_{1}\right)$. If a $C_{+}$characteristic emerges from the region $y<y_{1}$, i.e., $y_{+}\left(t=t_{0}\right)<y_{1}$, we know that $y_{+}(t)$ will decrease with time or, equivalently, $\xi_{+}(t)$ will increase with time and the $C_{+}$characteristic will not catch up with the shock front.

Equations (48)-(50) can also be solved numerically for different values of $k$. We plot the results for $k=5.5$ $\left(m_{1}=-1.77\right)$ and $k=6.5\left(m_{1}=-2.23\right)$ in Figure 1 . When $k_{c}<k<6$, the function $f(\xi)$ decreases with increasing $\xi$, while the function $g(\xi)$ increases with increasing $\xi$. This implies that when moving inward, away from the shock front, the pressure decreases while the Lorentz factor increases. For $k>6$, the situation is reversed. For all $k>k_{c}$, the function $h(\xi)$ increases with $\xi$, implying that the density always increases when moving inward, away from the shock front.

\section{PROPERTIES OF THE FLOW IN THE SELF-SIMILAR REGION}

We now examine the properties of the flow in the selfsimilar region bounded by $\xi=0$ and $\xi=\xi_{+}(t)$, where $\xi_{+}(t)$ coincides with a $C_{+}$characteristic that emerges from the region $y<y_{1}$. The evolution of the $C_{+}$characteristic is described by equation (70) with the initial condition $\xi_{+}\left(t=t_{0}\right)=\xi_{0}$ and, correspondingly, $y_{+}\left(t=t_{0}\right)=y_{0}<y_{1}$. By solving equation (70), we get the following equation for $y_{+}(t)$ :

$$
\left(\frac{y_{1}-y_{+}}{y_{1}-y_{0}}\right)^{\left(y_{2}-y_{1}\right) /\left(b-y_{1}\right)}\left(\frac{b-y_{+}}{b-y_{0}}\right)^{\left(b-y_{2}\right) /\left(b-y_{1}\right)}=\frac{t}{t_{0}} .
$$

We can now derive the asymptotic behavior of the $C_{+}$ characteristic at $t \rightarrow \infty$. In this limit $\left|y_{+}(t)\right|$ is large, and equation (71) yields

$$
y_{+}(t) \simeq-\alpha_{0} t
$$



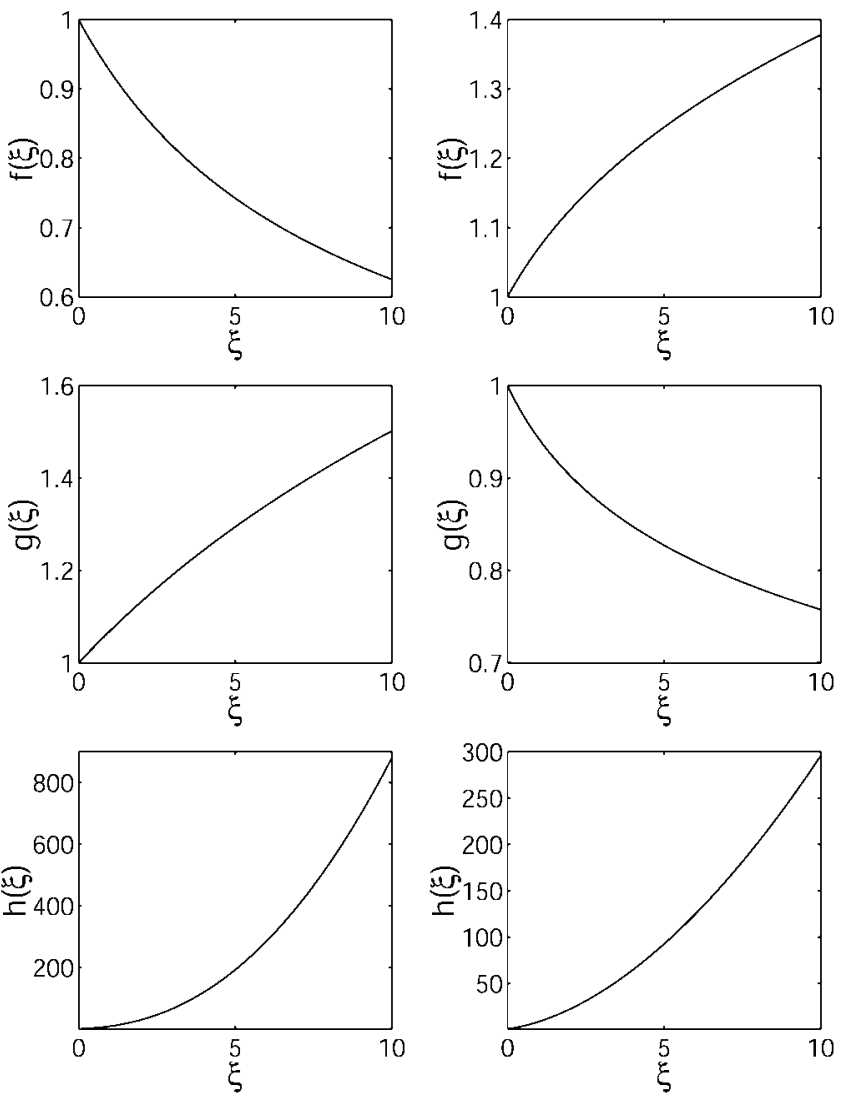

Fig. 1.-Distributions of the self-similar functions $f(\xi), g(\xi)$, and $h(\xi)$. The left-hand column corresponds to $k=5.5$ and $m_{1}=-1.77$, while the right-hand column corresponds to $k=6.5$ and $m_{1}=-2.23$.

where

$$
\alpha_{0}=\left(y_{1}-y_{0}\right)^{\left(y_{2}-y_{1}\right) /\left(b-y_{1}\right)}\left(b-y_{0}\right)^{\left(b-y_{2}\right) /\left(b-y_{1}\right)} / t_{0} .
$$

Substituting equation (63) into equation (72), we get

$$
\xi_{+}(t) \simeq \alpha_{+} t^{-\left(m_{1}+1\right)},
$$

where

$$
\alpha_{+}=\left(\frac{\alpha_{0}}{\alpha_{y}}\right)^{-\left(m_{1}+1\right)}
$$

The initial condition for the $C_{+}$characteristic, namely, the value of the $C_{+}$characteristic with which $\xi_{+}(t)$ coincides, contains the information about the initial explosion.

The shock front is accelerating with a power-law temporal dependence of its Lorentz factor, $\Gamma^{2} \propto t^{-m_{1}}$. How does the $C_{+}$characteristic propagate? Define $\gamma_{+}$as the Lorentz factor of the $C_{+}$characteristic. From equations (42), (61), and (74), we find that when $t \rightarrow \infty$,

$$
\gamma_{+}^{2}=(\sqrt{3}+2) \gamma^{2}\left(\xi_{+}\right)=(\sqrt{3}+2) \frac{1}{2} \Gamma^{2} g\left(\xi_{+}\right) \propto t^{2} .
$$

We see that irrespective of the value of $k$, the $C_{+}$characteristic always accelerates as $\gamma_{+}^{2} \propto t^{2}$. We can also calculate the thickness of the self-similar region. Using equations (5) and
(74), we obtain, when $t \rightarrow \infty$,

$$
R-r_{+}=\frac{R \xi_{+}}{\Gamma^{2}} \longrightarrow \text { const } .
$$

Note that when $-2<m_{1}<m_{1 c}$, the $C_{+}$characteristic accelerates faster than the shock front, but because the Lorentz factors of both surfaces are accelerating as power laws of time, the $C_{+}$characteristic can never catch up with the shock front. Instead, the distance between the two surfaces approaches a constant value at late times.

We can now examine the energy and mass contained in the self-similar region. The energy contained in the spherical shell between $\xi=0$ and $\xi=\xi_{+}(t)$ is given by

$E=\int_{r_{+}}^{R} 16 \pi p \gamma^{2} r^{2} d r=\frac{16 \pi}{3} w_{1} \Gamma^{2} R^{3} \int_{0}^{\xi_{+}} f(\xi) g(\xi) d \xi$.

Using equations (65) and (61), we can calculate the above integral for large values of $\xi_{+}$. This gives

$$
E \simeq \frac{16 \pi}{3}\left(-\frac{m_{1}+1}{m_{1}+k-3}\right) \alpha_{f} \alpha_{g} w_{1} \Gamma^{2} R^{3} \xi_{+}^{-\left(m_{1}+k-3\right) /\left(m_{1}+1\right)} .
$$

In deriving the above result we have used the fact that when $k>k_{c}$, we have $m_{1}+k-3>0$ and $m_{1}+1<0$, so that the exponent of $\xi_{+}$in the above equation, $-\left(m_{1}+k-3\right) /$ $\left(m_{1}+1\right)$, is positive. When $t \rightarrow \infty, \xi_{+}$is given by equation (74). In addition $\Gamma^{2} \propto t^{-m_{1}}$ and $w_{1}=\rho_{1} \propto R^{-k}$. Thus, when $t \rightarrow \infty$,

$$
E \longrightarrow \text { const }
$$

The total number of particles contained between $\xi=0$ and $\xi=\xi_{+}(t)$ is given by

$$
N=\int_{r_{+}}^{R} n^{\prime} 4 \pi r^{2} d r=8 \pi n_{1} R^{3} \int_{0}^{\xi_{+}} h(\xi) d \xi .
$$

Using equations (67) and (74), we obtain that for $t \rightarrow \infty$

$$
N \simeq 8 \pi\left(-\frac{m_{1}+1}{k-3}\right) \alpha_{h} n_{1} R^{3} \xi_{+}^{-(k-3) /\left(m_{1}+1\right)} \longrightarrow \text { const } .
$$

We have thus proved that both the energy and mass contained between the $C_{+}$characteristic and the shock front will approach constant values as $t \rightarrow \infty$. The situation is similar to the nonrelativistic case (Waxman \& Shvarts 1993). However, the mechanisms that cause the acceleration of the shock front are qualitatively different for the nonrelativistic and relativistic cases. In the nonrelativistic case, the kinetic energy contained in the self-similar region approaches a constant, while the thermal energy approaches zero as the radius of the shock front goes to infinity (Waxman \& Shvarts 1993). The acceleration of the shock front is not due to the conversion of thermal energy to kinetic energy. Rather, there is a continuous transfer of energy to new material shocked by the shock wave. The rate of this energy transfer is decreasing (to zero) with time, but the rate at which mass is overtaken by the shock decreases even faster. Thus, the shock accelerates. It is not that all the shell (i.e., the self-similar region) accelerates. Rather, a smaller and smaller fraction of the shell's mass is shocked to higher and higher velocity. The mass-averaged velocity of the shell approaches a constant. In the relativistic case, the 
acceleration is not concentrated only in a small fraction of the total mass of the shell. We can calculate the mass averaged Lorentz factor $\bar{\gamma}$ as follows:

$$
\begin{aligned}
\bar{\gamma} & =\frac{1}{N} \int_{r_{+}}^{R} \gamma n^{\prime} 4 \pi r^{2} d r \\
& =\frac{1}{N} \frac{8}{\sqrt{2}} \pi n_{1} R^{3} \Gamma \int_{0}^{\xi_{+}} h(\xi) g^{1 / 2}(\xi) d \xi,
\end{aligned}
$$

where $N$ is given by equation (81). Using equations (61), (67), (74), and (82), we obtain that for $t \rightarrow \infty$

$$
\bar{\gamma} \simeq \sqrt{2}\left(\frac{k-3}{m_{1}+2 k-4}\right) \alpha_{g}^{1 / 2} \Gamma \xi_{+}^{-\left(m_{1}+2\right) /\left[2\left(m_{1}+1\right)\right]} \propto t .
$$

Therefore, the whole self-similar region is accelerating. ${ }^{5}$ The total energy contained in the self-similar region will still approach a constant because the pressure in the self-similar region decreases with time as $p \propto t^{-k-m_{1}} f(\xi)$ (see eq. [20]). The acceleration of the shell is caused by the conversion of thermal energy to kinetic energy. The situation is similar to the accelerated expansion of a cloud of gas in vacuum. Indeed, from equation (77) we know that the thickness of the shell approaches a constant as $t \rightarrow \infty$, and since the radius of the shock front increases as $t$, the volume contained in the shell increases as $t^{2}$.

As pointed out by Johnson \& McKee (1971), postshock acceleration (the acceleration of the fluid after it is shocked) is important for a relativistic fluid. In a planar geometry, they estimated the final Lorentz factor of the shocked fluid to be $\gamma_{f} \simeq\left(\gamma_{s} / \sqrt{2}\right)^{1+\sqrt{3}}$, where $\gamma_{s}$ is the Lorentz factor of the shock front when the fluid is initially shocked. The importance of postshock acceleration was also demonstrated for transrelativistic blast waves in both planar and spherical geometries by Tan, Matzner, \& McKee (2001) using numerical simulations. They also derived approximate analytic expressions to describe transrelativistic blast waves. To see whether the self-similar solutions for steep power-law density profiles $\left(k>k_{c}\right)$ are consistent with the argument that postshock acceleration is important, we follow the evolution of a fluid element that is shocked at time $t=t_{0}$ by the ultrarelativistic spherical shock whose Lorentz factor is $\Gamma_{s}$ at that time. The evolution of the fluid element follows

$$
\frac{d r_{*}}{d t} \simeq 1-\frac{1}{2 \gamma_{*}^{2}}=1-\frac{1}{\Gamma^{2} g\left(\xi_{*}\right)},
$$

where we use the asterisk subscript to denote values associated with the fluid element. Combining the above equation with equation (44), we obtain

$$
t \frac{d \xi_{*}}{d t}=\frac{2-y_{*}}{2 g\left(\xi_{*}\right)},
$$

where

$$
y_{*}=\left[1+2\left(m_{1}+1\right) \xi_{*}\right] g\left(\xi_{*}\right) .
$$

\footnotetext{
${ }^{5}$ Note that when $-2<m_{1}<m_{1 c}$, the mass-averaged Lorentz factor $\bar{\gamma}$ increases with time faster than the shock Lorentz factor $\Gamma$. However, although the fluid behind the shock front accelerates faster than the shock front, it can never catch up with the shock front. This follows the same reasoning that explained the acceleration of the $C_{+}$characteristic; see the arguments below eq. (77)
}

Using equation (38), we can rewrite equation (86) as

$$
t \frac{d y_{*}}{d t}=\frac{\left(y_{*}-2\right)\left(y_{*}-b\right)}{y_{*}-y_{2}} .
$$

With the boundary condition $y_{*}\left(t=t_{0}\right)=1$, the above equation has the solution

$$
\left(2-y_{*}\right)^{\left(y_{2}-2\right) /(b-2)}\left(\frac{b-y_{*}}{b-1}\right)^{\left(b-y_{2}\right) /(b-2)}=\frac{t}{t_{0}} .
$$

The asymptotic behavior of the fluid element at large $t$ can be derived by setting $|y *(t)|$ to be large, and thus equation (89) yields

$$
y_{*}(t) \simeq-\alpha_{1} t,
$$

where

$$
\alpha_{1}=(b-1)^{\left(b-y_{2}\right) /(b-2)} / t_{0} .
$$

Substituting equation (63) into equation (90), we get

$$
\xi_{*}(t) \simeq \alpha_{*} t^{-\left(m_{1}+1\right)},
$$

where

$$
\alpha_{*}=\left(\frac{\alpha_{1}}{\alpha_{y}}\right)^{-\left(m_{1}+1\right)} .
$$

The Lorentz factor of the fluid element is described by

$$
\gamma_{*}^{2}=\frac{1}{2} \Gamma^{2} g\left(\xi_{*}\right)
$$

where $\Gamma$ is the Lorentz factor of the shock front at time $t$ and is related to $\Gamma_{s}$ by

$$
\frac{\Gamma^{2}}{\Gamma_{s}^{2}}=\left(\frac{t}{t_{0}}\right)^{-m_{1}} .
$$

Using equations (61), (92), and (95), we can rewrite equation (94) as

$$
\gamma_{*}^{2}=\frac{\Gamma_{s}^{2}}{2} t_{0}^{m_{1}} \alpha_{g} \alpha_{*}^{-\left(m_{1}+2\right) /\left(m_{1}+1\right)} t^{2} \propto t^{2} .
$$

Therefore, the fluid element is accelerating with $\gamma_{*} \propto t$. The acceleration of the fluid element is caused by the conversion of its internal energy to kinetic energy. When the available internal energy is exhausted, the acceleration stops. The time of this final stage can be estimated by setting the internal energy density of the fluid element equal to its rest energy density:

$$
e_{*}=n_{*} m_{p},
$$

where $n_{*}=n_{*}^{\prime} / \gamma_{*}$ and $e_{*}=3 p_{*}$ are the number density and energy density of the fluid element measured in the fluid frame, respectively. Using equations (20)-(22), we can rewrite equation (97) as

$$
\frac{\Gamma}{\sqrt{2}} g^{1 / 2}\left(\xi_{*}\right) f\left(\xi_{*}\right)=h\left(\xi_{*}\right) .
$$

For large $t$, we can substitute equations (61), (65), and (67) into the above equation and also use equations (92) and (95) to obtain

$$
t_{f}=\frac{\Gamma_{s}}{\sqrt{2}} t_{0}^{m_{1} / 2} \alpha_{g}^{1 / 2} \alpha_{f} \alpha_{h}^{-1} \alpha_{*}^{-\left(m_{1}-2\right) /\left[2\left(m_{1}+1\right)\right]},
$$

where $t_{f}$ is the final time when the fluid element stops accelerating. The final Lorentz factor of the fluid element can be 
calculated by plugging the above equation into equation (96). We get

$$
\gamma_{f}=\frac{\Gamma_{s}^{2}}{2} \alpha_{2}
$$

where

$$
\begin{aligned}
\alpha_{2} & =\alpha_{g} \alpha_{f} \alpha_{h}^{-1} \alpha_{y}^{-m_{1}}(b-1)^{m_{1}\left(b-y_{2}\right) /(b-2)} \\
& =\left[2 \sqrt{3}\left(k-k_{c}\right)\right]^{(2 \sqrt{3} / 3-1)(k-6) /[k-(5-\sqrt{3} / 3)]} .
\end{aligned}
$$

Here $\alpha_{2}$ is an increasing function of $k$. As $k$ increases from $k_{c}$ to $6, \alpha_{2}$ increases from 0 to 1 . For $k>k_{c}, \alpha_{2}$ increases very slowly as $k$ increases. For example, when $k=20$, $\alpha_{2}=1.74$. From equation (100) we see that the final Lorentz factor of the fluid element is of the order of $\left(\Gamma_{s} / \sqrt{2}\right)^{2}$ (except for the limiting case in which $k$ is very close to $k_{c}$ ). We have therefore shown that postshock acceleration is also very important for the self-similar solutions in a steep power-law density profile. However, because of the spherical geometry in this case, the postshock acceleration is not as strong as that in the planar geometry case, where the final Lorentz factor $\gamma_{f}$ reaches $\sim\left(\gamma_{s} / \sqrt{2}\right)^{1+\sqrt{3}}$ (Johnson \& McKee 1971). ${ }^{6}$

\section{APPROXIMATE (ANALYTIC) STABILITY ANALYSIS}

In order to analyze the stability of the self-similar solutions obtained in $\S 2.2$, we first follow an analytic approach. The perturbations of greatest interest are those that grow fast compared to the acceleration of the unperturbed Lorentz factor of the shock front $\Gamma_{0}$. For these perturbations, we may assume that $\Gamma_{0}$ is nearly a constant during the growth of the perturbation and thus simplify the equations by separating variables. As explained later, these assumptions limit the generality of our results, and so in $\S 5$ we relax the above approximation and use numerical simulations to exactly solve the evolution of the perturbations. The numerical simulations demonstrate that the results obtained based on this simplified analytic approach are qualitatively valid.

\subsection{Derivation of Linear Perturbation Equations}

For the analytic approach to the stability analysis of the self-similar solutions, we use linear perturbation analysis similar to that used in the nonrelativistic case (Sari et al. 2000). We start from the equations of motion for an ideal relativistic fluid:

$$
\begin{gathered}
\frac{\partial}{\partial t}\left(n^{\prime}\right)+\nabla \cdot\left(n^{\prime} \boldsymbol{v}\right)=0, \\
\gamma^{2}(e+p)\left[\frac{\partial \boldsymbol{v}}{\partial t}+(\boldsymbol{v} \cdot \boldsymbol{\nabla}) \boldsymbol{v}\right]+\left(\boldsymbol{\nabla} p+\boldsymbol{v} \frac{\partial p}{\partial t}\right)=0, \\
\frac{\partial}{\partial t}\left(\frac{\gamma^{4 / 3} p}{n^{1 / 3}}\right)+(\boldsymbol{v} \cdot \boldsymbol{\nabla})\left(\frac{\gamma^{4 / 3} p}{n^{1 / 3}}\right)=0,
\end{gathered}
$$

where $v$ and $\gamma$ are the fluid velocity and Lorentz factor, respectively, in the perturbed solution measured in the laboratory frame, $e$ and $p$ are the energy density and pressure, respectively, in the perturbed solution measured in the fluid frame, and $n^{\prime}$ is the fluid density in the perturbed solution measured in the laboratory frame. We use the Eulerian

\footnotetext{
${ }^{6}$ For a discussion of the difference between planar and spherical geometries on postshock acceleration, see Tan et al. (2001).
}

perturbation approach; i.e., the perturbed quantities are defined as the difference between the perturbed solution and the unperturbed one in the same spatial point. Therefore, we define the perturbed hydrodynamic quantities as

$$
\begin{gathered}
\delta p(r, \theta, \phi, t)=p(r, \theta, \phi, t)-p_{0}(r, t), \\
\delta \boldsymbol{v}(r, \theta, \phi, t)=\boldsymbol{v}(r, \theta, \phi, t)-v_{0}(r, t) \hat{\boldsymbol{r}}=\delta v_{r} \hat{\boldsymbol{r}}+\delta \boldsymbol{v}_{T}, \\
\delta n^{\prime}(r, \theta, \phi, t)=n^{\prime}(r, \theta, \phi, t)-n_{0}^{\prime}(r, t),
\end{gathered}
$$

where the quantities with subscript " 0 " are the unperturbed values. Substituting the above quantities into equations (102)-(104), we obtain the following linear perturbation equations:

$$
\begin{gathered}
\frac{\partial}{\partial t} \delta n^{\prime}+\frac{1}{r^{2}} \frac{\partial}{\partial r}\left\{r ^ { 2 } \left[\frac{1}{2 \gamma_{0}^{4}}\left(1+\frac{1}{2 \gamma_{0}^{2}}\right) n_{0}^{\prime} \delta \gamma^{2}\right.\right. \\
\left.\left.+\left(1-\frac{1}{2 \gamma_{0}^{2}}\right) \delta n^{\prime}\right]\right\}+n_{0}^{\prime} \nabla_{T} \cdot \delta \boldsymbol{v}_{T}=0 \\
\frac{2}{\gamma_{0}^{4}}\left[\left(1+\frac{1}{2 \gamma_{0}^{2}}\right) \frac{\partial \gamma_{0}^{2}}{\partial t}+\frac{\partial \gamma_{0}^{2}}{\partial r}\right]\left(\gamma_{0}^{2} \delta p+p_{0} \delta \gamma^{2}\right) \\
+4 \gamma_{0}^{2} p_{0}\left\{\frac{\partial}{\partial t}\left[\frac{1}{2 \gamma_{0}^{4}}\left(1+\frac{1}{2 \gamma_{0}^{2}}\right) \delta \gamma^{2}\right]\right. \\
+\left(1-\frac{1}{2 \gamma_{0}^{2}}\right) \frac{\partial}{\partial r}\left[\frac{1}{2 \gamma_{0}^{4}}\left(1+\frac{1}{2 \gamma_{0}^{2}}\right) \delta \gamma^{2}\right] \\
+\frac{1}{4 \gamma_{0}^{8}}\left(1+\frac{1}{\gamma_{0}^{2}}\right)\left(\frac{\partial \gamma_{0}^{2}}{\partial r}\right) \delta \gamma^{2} \\
+\frac{1}{2 \gamma_{0}^{4}}\left(1+\frac{1}{2 \gamma_{0}^{2}}\right)\left(\frac{\partial p_{0}}{\partial t}\right) \delta \gamma^{2}=0 \\
+\frac{1}{2 \gamma_{0}^{4}}\left(1+\frac{1}{2 \gamma_{0}^{2}}\right) \frac{\partial}{\partial r}\left(\frac{\gamma_{0}^{4 / 3} p_{0}}{n_{0}^{4 / 3}}\right) \delta \gamma^{2}=0 \\
4 p_{0} \gamma_{0}^{2}\left[\frac{\partial}{\partial t} \delta \boldsymbol{v}_{T}+\left(1-\frac{1}{2 \gamma_{0}^{2}}\right) \frac{\partial}{\partial r} \delta \boldsymbol{v}_{T}\right. \\
\left.+\left(1-\frac{1}{2 \gamma_{0}^{2}}\right) \frac{1}{r} \delta \boldsymbol{v}_{T}\right]+\nabla_{T} \delta p+\left(\frac{\partial p_{0}}{\partial t}\right) \delta \boldsymbol{v}_{T}=0 \\
\frac{\partial}{\partial t}\left[\frac{\gamma_{0}^{4 / 3} p_{0}}{n_{0}^{\prime 4 / 3}}\left(\frac{2}{3} \frac{\delta \gamma^{2}}{\gamma_{0}^{2}}+\frac{\delta p}{p_{0}}-\frac{4}{3} \frac{\delta n^{\prime}}{n_{0}^{\prime}}\right)\right] \\
+\left(1-\frac{1}{2 \gamma_{0}^{2}}\right) \frac{\partial}{\partial r}\left[\frac{\gamma_{0}^{4 / 3} p_{0}}{n_{0}^{\prime 4 / 3}}\left(\frac{2}{3} \frac{\delta \gamma^{2}}{\gamma_{0}^{2}}+\frac{\delta p}{p_{0}}-\frac{4}{n_{0}^{\prime}}\right)\right]
\end{gathered}
$$

where we have used the relations

$$
\begin{gathered}
\frac{\partial v_{0}}{\partial t}=\frac{1}{2 \gamma_{0}^{4}}\left(1+\frac{1}{2 \gamma_{0}^{2}}\right) \frac{\partial \gamma_{0}^{2}}{\partial t}, \\
\frac{\partial v_{0}}{\partial r}=\frac{1}{2 \gamma_{0}^{4}}\left(1+\frac{1}{2 \gamma_{0}^{2}}\right) \frac{\partial \gamma_{0}^{2}}{\partial r}, \\
\delta v_{r}=\frac{1}{2 \gamma_{0}^{4}}\left(1+\frac{1}{2 \gamma_{0}^{2}}\right) \delta \gamma^{2},
\end{gathered}
$$


and the operator $\boldsymbol{\nabla}_{T} \equiv(\hat{\theta} / r)(\partial / \partial \theta)+(\hat{\phi} / r)(\partial / \partial \phi)$ acts as follows on a scalar $\Psi$ and a vector $f$ :

$$
\begin{gathered}
\nabla_{T} \Psi=\frac{1}{r} \frac{\partial \Psi}{\partial \theta} \hat{\theta}+\frac{1}{r \sin \theta} \frac{\partial \Psi}{\partial \phi} \hat{\phi}, \\
\nabla_{T} \cdot \boldsymbol{f}=\frac{1}{r \sin \theta} \frac{\partial}{\partial \theta}\left(\sin \theta f_{\theta}\right)+\frac{1}{r \sin \theta} \frac{\partial f_{\phi}}{\partial \phi} .
\end{gathered}
$$

Since the unperturbed quantities satisfy equations (20)(22), we write

$$
\begin{gathered}
p_{0}=\frac{2}{3} \rho_{1} \Gamma_{0}^{2} f(\xi), \\
\gamma_{0}^{2}=\frac{1}{2} \Gamma_{0}^{2} g(\xi), \\
n_{0}^{\prime}=2 n_{1} \Gamma_{0}^{2} h(\xi),
\end{gathered}
$$

where $\Gamma_{0}$ is the unperturbed Lorentz factor of the shock front and $\xi$ is the similarity variable, defined as

$$
\xi=\left(1-\frac{r}{R_{0}}\right) \Gamma_{0}^{2},
$$

where $R_{0}$ is the unperturbed radius of the shock front. We further define the perturbation variables as

$$
\begin{array}{r}
\delta \gamma^{2}(r, \theta, \phi, t)=\frac{1}{2} \Gamma_{0}^{2} \delta g(\xi) Y_{l m}(\theta, \phi) X(t), \\
\delta \boldsymbol{v}_{T}(r, \theta, \phi, t)=-\frac{1}{\Gamma_{0}^{2}} \delta v_{T}(\xi) \tilde{\boldsymbol{\nabla}}_{T} Y_{l m}(\theta, \phi) X(t), \\
\delta p(r, \theta, \phi, t)=\frac{2}{3} \rho_{1} \Gamma_{0}^{2} \delta f(\xi) Y_{l m}(\theta, \phi) X(t), \\
\delta n^{\prime}(r, \theta, \phi, t)=2 n_{1} \Gamma_{0}^{2} \delta h(\xi) Y_{l m}(\theta, \phi) X(t),
\end{array}
$$

where the operator $\tilde{\mathbf{\nabla}}_{T} \equiv \hat{\theta}(\partial / \partial \theta)+\hat{\phi}(1 / \sin \theta)(\partial / \partial \phi)$. Note that the variables $\xi$ and $t$ are separated in above definitions of the perturbations, and so we consider only "global" perturbations (Sari et al. 2000; Cox 1980). The function $X(t)$ measures the amplitude of the perturbation relative to the unperturbed values.

Substituting equations (117)-(119) and (121)-(124) into equations (108)-(111), we obtain

$$
\begin{gathered}
{\left[q+2-k-m_{1}+\frac{2\left(m_{1}+2\right)}{\left(y-y_{2}\right)}\right] \frac{\delta h}{h}-\frac{(y-2)}{2}\left(\frac{1}{g} \frac{1}{h} \frac{d \delta h}{d \xi}\right)} \\
+\left[\frac{-4\left(m_{1}+2\right)}{\left(y-y_{2}\right)}+\frac{2\left(m_{1}+k-2\right)(y-d)}{\left(y-y_{2}\right)(y-2)}\right] \frac{\delta g}{g} \\
-\left(\frac{1}{g} \frac{1}{g} \frac{d \delta g}{d \xi}\right)+\frac{l(l+1)}{\Gamma_{0}^{2}} \delta v_{T}=0, \\
{\left[-k-3 m_{1}+q+\frac{2\left(m_{1}+2\right)(y-2)}{\left(y-y_{2}\right)}\right] \frac{\delta f}{f}} \\
-\frac{(y+2)}{2}\left(\frac{1}{g} \frac{1}{f} \frac{d \delta f}{d \xi}\right) \\
+\left[2 q-\frac{2\left(m_{1}+2\right)(y-4)}{\left(y-y_{2}\right)}-\frac{2\left(m_{1}+k-4\right)}{\left(y-y_{2}\right)}\right] \frac{\delta g}{g} \\
-(y-2)\left(\frac{1}{g} \frac{1}{g} \frac{d \delta g}{d \xi}\right)=0,
\end{gathered}
$$

$$
\begin{aligned}
& {\left[2\left(m_{1}+q+1\right)-\frac{2\left(m_{1}+k-4\right)}{\left(y-y_{2}\right)}\right] g \delta v_{T}} \\
& -(y-2) \frac{d \delta v_{T}}{d \xi}-\frac{\delta f}{f}=0, \quad(127) \\
& {\left[\frac{2}{3} q-\frac{2}{3} \frac{\left(m_{1}+2\right)(y-4)}{\left(y-y_{2}\right)}+\frac{2\left(m_{1}+k-4\right)}{\left(y-y_{2}\right)}\right.} \\
& \left.-\frac{8}{3} \frac{\left(m_{1}+k-2\right)(y-d)}{\left(y-y_{2}\right)(y-2)}\right] \frac{\delta g}{g}-\frac{(y-2)}{3}\left(\frac{1}{g} \frac{1}{g} \frac{d \delta g}{d \xi}\right) \\
& +\left[q-\frac{\left(m_{1}+k-4\right)(y-2)}{\left(y-y_{2}\right)}\right] \frac{\delta f}{f}-\frac{(y-2)}{2}\left(\frac{1}{g} \frac{1}{f} \frac{d \delta f}{d \xi}\right) \\
& -\frac{4}{3}\left[q-\frac{\left(m_{1}+k-2\right)(y-d)}{\left(y-y_{2}\right)}\right] \frac{\delta h}{h} \\
& +\frac{2}{3}(y-2)\left(\frac{1}{g} \frac{1}{h} \frac{d \delta h}{d \xi}\right)=0,
\end{aligned}
$$

where we have used equations (48)-(50) and the following relations:

$$
\begin{gathered}
\frac{d \Gamma_{0}^{2}}{d t}=-m_{1} \frac{\Gamma_{0}^{2}}{t}, \\
\frac{\partial \xi}{\partial r}=-\frac{1}{t}\left[\Gamma_{0}^{2}+\frac{1}{2\left(m_{1}+1\right)}\right], \\
\frac{\partial \xi}{\partial t}=\frac{1}{t}\left[\Gamma_{0}^{2}-\frac{m_{1}}{2\left(m_{1}+1\right)}-\xi\left(m_{1}+1\right)\right], \\
\frac{d \rho_{1}}{d t}=-k \frac{\rho_{1}}{t}\left[1-\frac{m_{1}}{2\left(m_{1}+1\right) \Gamma_{0}^{2}}\right], \\
\frac{d n_{1}}{d t}=-k \frac{n_{1}}{t}\left[1-\frac{m_{1}}{2\left(m_{1}+1\right) \Gamma_{0}^{2}}\right], \\
r=t\left[1-\frac{1}{2\left(m_{1}+1\right) \Gamma_{0}^{2}}-\frac{\xi}{\Gamma_{0}^{2}}\right] .
\end{gathered}
$$

Note that in deriving equations (125)-(128) we have assumed that there is no perturbation in the external medium. Moreover, in order to separate variables, $X(t)$ has to be a power law in time, $X(t) \propto t^{q}$, where $q$ defines the temporal evolution of the perturbation amplitude. If the real part of $q(\operatorname{Re}[q])$ is positive, then the perturbation grows, while if the real part of $q$ is negative, then the perturbation decays.

In equation (125), the term $l(l+1) / \Gamma_{0}^{2}$ is associated with causality, namely, the fact that a perturbation can only propagate at a speed $\lesssim c / \Gamma_{0}$ in the transverse direction and hence expand across a maximum opening angle of $\sim 1 / \Gamma_{0}$. Since $\Gamma_{0}$ is a function of time, it is not possible to achieve a complete separation of variables for this equation, in contrast with the nonrelativistic case. However, for any constant value of $\Gamma_{0}$, we can still calculate the power-law index for the growth of the perturbation, $q$. These results are meaningful if we find $\operatorname{Re}[q]>\left|m_{1}\right|$, so that perturbations grow on a timescale shorter than the timescale for changes in $\Gamma_{0}$. Therefore, the assumptions of variable separation and fixed $\Gamma_{0}$ limit the generality of the results. However, even if we find $\operatorname{Re}[q]<\left|m_{1}\right|$, we should still be able to gain an insight into some qualitative properties of the perturbation amplitude evolution.

Also note that for a perturbation with a fixed wavenumber $l$, there is a qualitative change when $\Gamma_{0} \simeq l$, due to 
causality, and hence self-similarity breaks. This is reflected in the term $l(l+1) / \Gamma_{0}^{2}$ in equation (125).

Equations (125)-(128) are a complete set of first-order differential equations for $\delta f, \delta g, \delta h$, and $\delta v_{T}$. After some algebraic manipulations, one may write the equations for the first-order terms $d \delta f / d \xi, d \delta g / d \xi, d \delta h / d \xi$, and $d \delta v_{T} / d \xi$ in the following matrix form:

$$
\begin{aligned}
\left(y^{2}-8 y+4\right) \frac{d}{d \xi}\left(\begin{array}{c}
\delta f \\
\delta g \\
\delta h \\
\delta v_{T}
\end{array}\right) \\
=\mathbf{A}\left[q, k, l(l+1) / \Gamma_{0}^{2}, \xi\right]\left(\begin{array}{c}
\delta f \\
\delta g \\
\delta h \\
\delta v_{T}
\end{array}\right),
\end{aligned}
$$

where $\mathbf{A}$ is a $4 \times 4$ matrix. Note that $\left(y^{2}-8 y+4\right)=\left(y-y_{1}\right)\left(y-y_{2}\right)$. Thus, the solutions for the perturbation variables must pass the same singular point (or the sonic line), $y_{1}=4-2 \sqrt{3}$, as the unperturbed variables. Therefore, the value of $q$ can be found by requiring that the solutions pass through the singular point $y_{1}$. This is very similar to the nonrelativistic case (Sari et al. 2000).

In order to numerically integrate the differential equations (135) and derive $q$, we need to specify the boundary conditions at the shock front when the shock is perturbed. Since the relativistic jump conditions across the shock front must be satisfied, we have

$$
\begin{aligned}
& p=\frac{2}{3} \Gamma^{2} \rho_{1}, \\
& n^{\prime}=2 \Gamma^{2} n_{1}, \\
& \gamma^{2}=\frac{1}{2} \Gamma^{2},
\end{aligned}
$$

where $\Gamma$ is the Lorentz factor of the perturbed shock front. By linearizing these boundary conditions with respect to the perturbed quantities, we find

$$
\begin{gathered}
\delta p+\left(\frac{\partial p_{0}}{\partial r}\right) \delta R=\frac{4}{3} \Gamma_{0}^{4} \rho_{1} \frac{d}{d t} \delta R-\frac{2 k}{3} \Gamma_{0}^{2} \rho_{1} \frac{\delta R}{R_{0}}, \\
\delta n^{\prime}+\left(\frac{\partial n_{0}^{\prime}}{\partial r}\right) \delta R=4 \Gamma_{0}^{4} n_{1} \frac{d}{d t} \delta R-2 k \Gamma_{0}^{2} n_{1} \frac{\delta R}{R_{0}}, \\
\delta \gamma^{2}+\left(\frac{\partial \gamma_{0}^{2}}{\partial r}\right) \delta R=\Gamma_{0}^{4} \frac{d}{d t} \delta R,
\end{gathered}
$$

where $\delta R$ is the deviation of the perturbed shock radius $R$ from the unperturbed shock radius $R_{0}$. In deriving equations (139)-(141), we used the relations $\rho_{1} \propto R^{-k}$ and

$$
\delta \Gamma^{2}=2 \Gamma_{0}^{4} \frac{d}{d t} \delta R,
$$

where $\delta \Gamma^{2}$ is the deviation of the square of the perturbed shock Lorentz factor $\Gamma^{2}$ from the square of the unperturbed shock Lorentz factor $\Gamma_{0}^{2}$.

We now define

$$
\delta R(\theta, \phi, t)=\eta \frac{1}{\Gamma_{0}^{2}} R_{0} Y_{l m}(\theta, \phi) X(t),
$$

where $\eta$ is a scale factor that can have an arbitrary value; for convenience we set $\eta=1$. Substituting equations (143), (117)-(119), (121), (123), and (124) into equations (139)-(141), we find

$$
\begin{aligned}
& \delta f=\frac{d f}{d \xi}+2(m+q+1), \\
& \delta h=\frac{d h}{d \xi}+2(m+q+1), \\
& \delta g=\frac{d g}{d \xi}+2(m+q+1) .
\end{aligned}
$$

Another boundary condition results from the requirement that the tangential velocities must be continuous across the shock front, yielding

$$
\delta \boldsymbol{v}_{T}=-\frac{1}{R_{0}} \tilde{\mathbf{\nabla}}_{T} \delta R .
$$

Substituting equations (122) and (143) into this equation, we get

$$
\delta v_{T}=1 .
$$

Note that here $\delta v_{T}=1$ does not mean that the tangential velocity is equal to the speed of light, but rather that it is normalized by the definition of $\delta v_{T}$ in equation (122) with the scaling factor of $1 / \Gamma_{0}^{2}$. Equations (144)-(146) and (148) are the four boundary conditions necessary to solve the perturbation equations.

\subsection{Numerical Results}

Based on the derivations presented in the previous subsection, we may now examine the stability of different modes for different values of $k$. As a particular example, we consider the case of $k=5.5$. We derive $q$ for different values of the mode wavenumber $l$ by integrating the differential equation (135) from the shock front to its interior and requiring that the solutions pass through the singular point. The results are shown in Figure 2. The top panel shows the real component of $q(\operatorname{Re}[q])$ as a function of $[l(l+1)]^{1 / 2} / \Gamma_{0}$, where $\Gamma_{0}$ is treated as a scaling factor. As mentioned before, $\operatorname{Re}[q]$ determines the growth rate of the perturbation. In the bottom panel, we plot the imaginary component of $q$ $(\operatorname{Im}[q])$, which provides the oscillation frequency of the perturbation. Figure 2 separates the behavior of $q$ into three different regimes:

1. In the regime of small $l / \Gamma_{0}\left(0<l / \Gamma_{0}<0.87\right), q$ is a real number and $\operatorname{Re}[q]$ is positive, implying that the perturbation grows monotonically in time. The value of $\operatorname{Re}[q]$ increases as $l$ increases. Note that $q$ vanishes in the limit of $l \rightarrow 0$. This result can be derived analytically by comparing two unperturbed spherical solutions with different parameters.

2 . In the regime of intermediate $l / \Gamma_{0}\left(0.87<l / \Gamma_{0}<17\right)$, $q$ is a complex number and $\operatorname{Re}[q]$ is positive, implying that the perturbation grows while oscillating. As $l$ increases, the real part $\operatorname{Re}[q]$ decreases, while the imaginary part $\operatorname{Im}[q]$ increases. Note that the transition between the real and imaginary solutions for $q$ occurs at $l / \Gamma_{0} \sim 0.87$. This result follows from causality. When the wavelength of the perturbation $(\sim 1 / l)$ is smaller than $1 / \Gamma_{0}$, the maximum angular separation of two regions that can interact with each other, the perturbation can oscillate. 

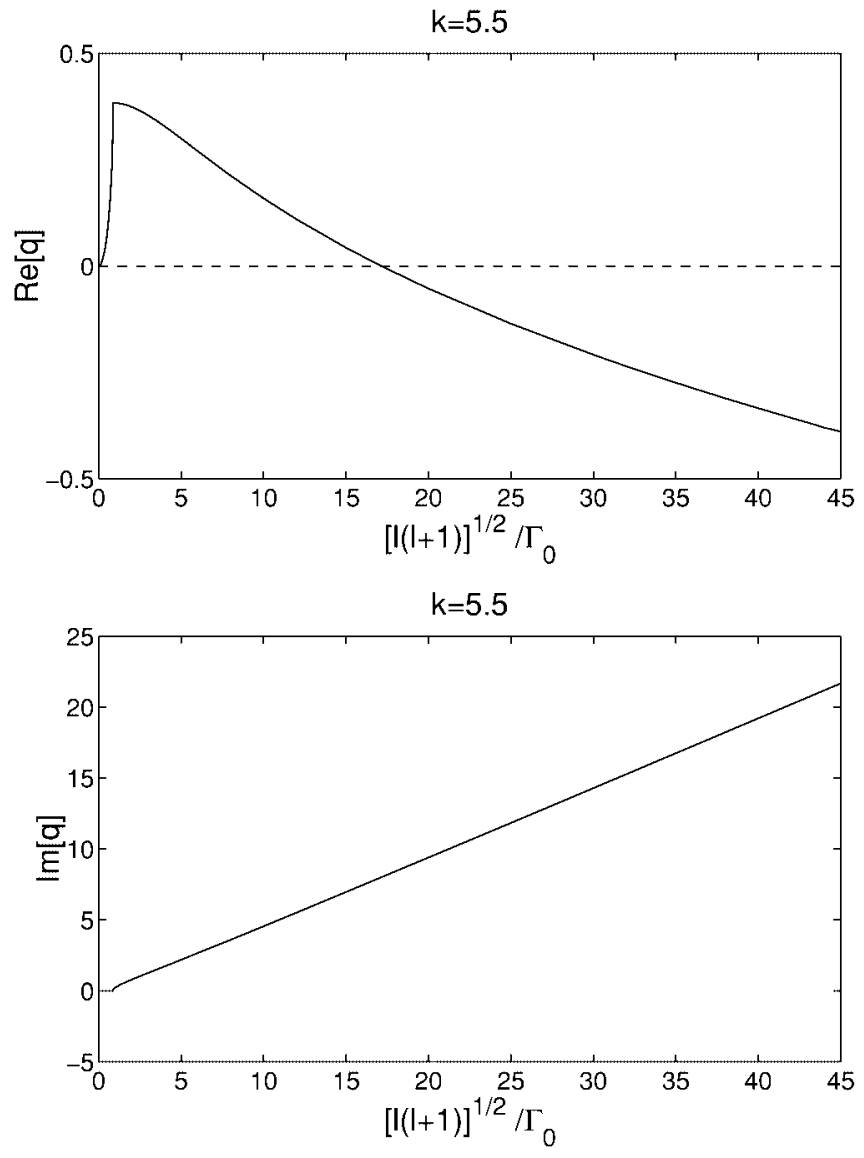

FIG. 2.-Perturbation growth rate, $q$, as a function of $[l(l+1)]^{1 / 2} / \Gamma_{0}$. The top and bottom panels show $\operatorname{Re}[q]$ and $\operatorname{Im}[q]$, respectively.

3. Finally, in the regime of large $l / \Gamma_{0}\left(l / \Gamma_{0}>17\right), q$ is a complex number and $\operatorname{Re}[q]$ is negative, implying that the perturbation decays while oscillating. The value of $\operatorname{Re}[q]$ decreases (so the absolute value of $\operatorname{Re}[q]$ increases) as $l$ increases, while the value of $\operatorname{Im}[q]$ increases as $l$ increases.

The actual evolution of a perturbation is shaped by the fact that $\Gamma_{0}$ increases with time as $\Gamma_{0}^{2} \propto t^{-m_{1}}$. If initially the wavenumber of the perturbation is sufficiently large so that it is in the regime of large $l / \Gamma_{0}$, the perturbation will start to decay while oscillating. As time progresses, $l / \Gamma_{0}$ decreases, and so both $|\operatorname{Re}[q]|$ and $\operatorname{Im}[q]$ decrease, the perturbation decays with slower speed, and oscillates on longer timescales. As soon as the perturbation enters the regime of intermediate $l / \Gamma_{0}$, it starts to grow slowly over time and oscillate on even longer timescales. The growth rate slowly increases over time, but is always limited by the rather small upper bound, $\operatorname{Re}[q] \lesssim 0.38$. Eventually, the perturbation enters the regime of small $l / \Gamma_{0}$ and grows slowly without oscillating. As $t$ increases, the growth rate approaches zero, and so the perturbation saturates. Therefore, perturbations with large wavenumbers (short wavelengths) grow when $1 \lesssim l / \Gamma_{0} \lesssim 10$ only by a modest factor. In the case of intermediate wavenumbers, the perturbation goes through the two regimes of intermediate and small $l / \Gamma_{0}$. Therefore, it grows slowly with some initial oscillations, but soon afterward it stops oscillating and saturates. Perturbations with small wavenumbers stay in the regime of small $l / \Gamma_{0}$. The perturbation grows slowly without oscillating at the beginning but soon saturates.
The above results remain qualitatively the same for all values of $k>4.134$.

\section{NUMERICAL SIMULATIONS}

In our approximate stability analysis of $\S 4$, we were considering perturbations that grow fast compared to changes in $\Gamma_{0}$ (i.e., $\operatorname{Re}[q]>\left|m_{1}\right|$ ), and so we were able to separate variables based on the assumption of a constant $\Gamma_{0}$. We derived some qualitative results for the evolution of the perturbations, which are also suitable for the case of $\operatorname{Re}[q]<\left|m_{1}\right|$. In this section, we relax the above approximations and verify those qualitative results by a direct integration of the partial different equations that determine the evolution of the perturbation variables, without assuming separability of the solutions with respect to $\xi$ and $t$.

Instead of equations (121)-(124), we redefined the perturbation variables as

$$
\begin{array}{r}
\delta \gamma^{2}(r, \theta, \phi, t)=\frac{1}{2} \Gamma_{0}^{2} \delta g(\xi, t) Y_{l m}(\theta, \phi), \\
\delta \boldsymbol{v}_{T}(r, \theta, \phi, t)=-\frac{1}{\Gamma_{0}^{2}} \delta v_{T}(\xi, t) \tilde{\mathbf{\nabla}}_{T} Y_{l m}(\theta, \phi), \\
\delta p(r, \theta, \phi, t)=\frac{2}{3} \rho_{1} \Gamma_{0}^{2} \delta f(\xi, t) Y_{l m}(\theta, \phi), \\
\delta n^{\prime}(r, \theta, \phi, t)=2 n_{1} \Gamma_{0}^{2} \delta h(\xi, t) Y_{l m}(\theta, \phi) .
\end{array}
$$

Equations (125)-(128) were then replaced by four partial differential equations (PDEs) for the perturbation variables $\delta f(\xi, t), \delta g(\xi, t), \delta h,(\xi, t)$, and $\delta v_{T}(\xi, t)$. We then solved for the evolution of these perturbation variables by numerically integrating the PDEs with appropriate initial values. In our numerical simulations, the outer boundary $(\xi=0)$ is the shock front where the shock jump conditions are assumed to be satisfied. We can still define $\delta R$ as in equation (143). Then at the outer boundary the perturbation variables satisfy

$$
\begin{gathered}
\delta f(0, t)=\frac{d f}{d \xi} X(t)+2\left(m_{1}+1\right) X(t)+2 t \frac{d X(t)}{d t}, \\
\delta h(0, t)=\frac{d h}{d \xi} X(t)+2\left(m_{1}+1\right) X(t)+2 t \frac{d X(t)}{d t}, \\
\delta g(0, t)=\frac{d g}{d \xi} X(t)+2\left(m_{1}+1\right) X(t)+2 t \frac{d X(t)}{d t}, \\
\delta v_{T}(0, t)=X(t) .
\end{gathered}
$$

The inner boundary is chosen to be sufficiently large so as to cover the entire similarity region that is bounded by a inner $C_{+}$characteristic. This way, the values of the perturbation variables at the inner boundary cannot affect the shock front.

The numerical simulations gave us the same behavior for the perturbations as the previously mentioned analytical results for the growth rate $q$. In Figure 3, we show the evolution of $X(t)$ in a numerical simulation with $k=5.5$ and $l / \Gamma_{0}=75$. We plot $X(t)$ in the range $t \in[1,10]$ in the top panel and $t \in[1,100]$ in the bottom panel. Note that $X(t)$ describes the relative displacement of the perturbed shock radius from the unperturbed value, and we set its initial value to be $X(t=1)=1.0$. From Figure 3 we see that $X$ oscillates over time over increasingly longer timescales and stops oscillating at late times. Its amplitude first decreases, 

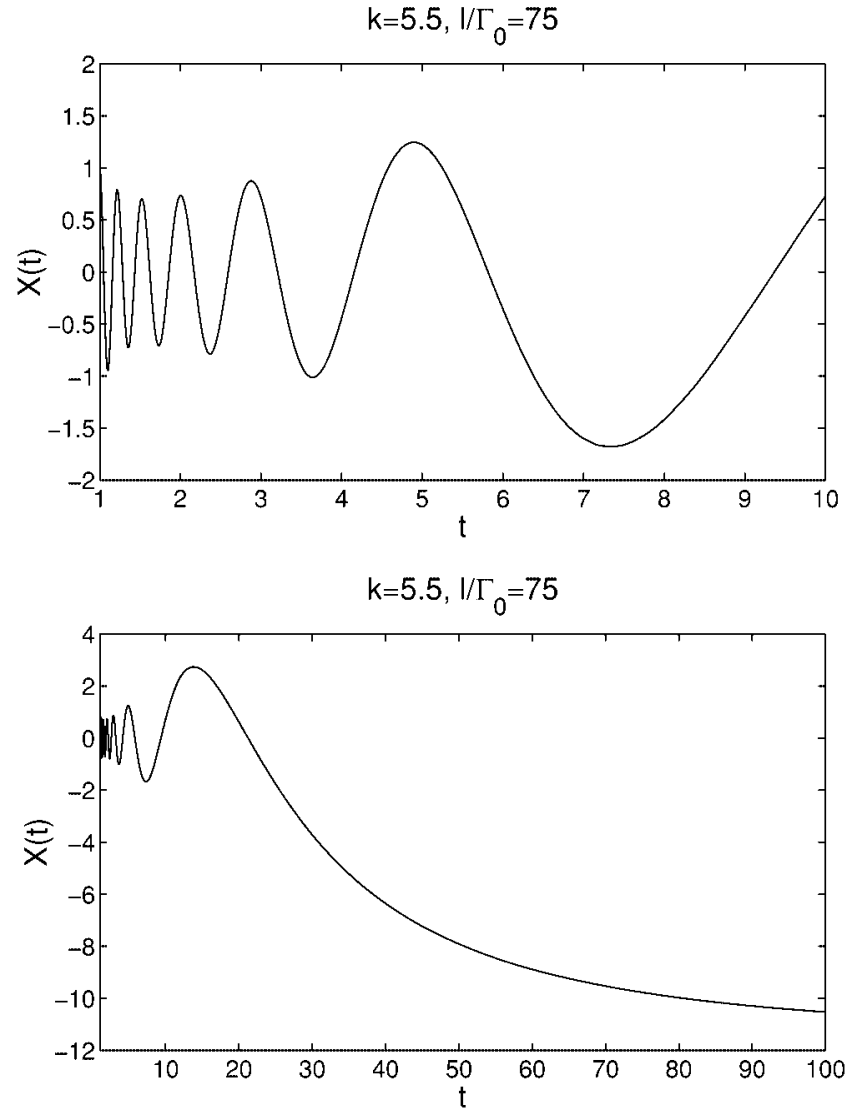

Fig. 3.-Evolution of $X(t)$ for $k=5.5$ and $l / \Gamma_{0}=75$. The top and bottom panels show $t \in[1,10]$ and $t \in[1,100]$, respectively.

then slowly increases, and finally saturates. Overall, it grows by a factor of $\sim 10$. These results are consistent with the previous discussion of the three regimes for the evolution of the perturbations.

\section{CONCLUSIONS}

We have derived the self-similar solutions for an ultrarelativistic blast wave in an external medium with a density profile $\rho_{1} \propto r^{-k}$ and $k>4$. The solutions exist for $k$ larger than a critical value $k_{c}=4.134$. They describe the flow in the self-similar region bounded by the shock front and a $C_{+}$ characteristic. The shock front accelerates with Lorentz factor $\Gamma^{2} \propto t^{-m_{1}}$ and $m_{1}<-1.134$, while the $C_{+}$characteristic accelerates with Lorentz factor $\gamma^{2} \propto t^{2}$. The energy and mass contained inside the self-similar region approach constant values as time diverges.

We have found that at large wavenumbers the perturbations first decay, then grow slowly over time, and eventually saturate. The initial decay and the intermediate growth are accompanied by temporal oscillations. These small wavelength perturbations grow when $1 \lesssim l / \Gamma_{0} \lesssim 10$, with an overall factor of $\sim 10$. At intermediate wavenumbers, the perturbations first grow slowly and then saturate. The initial growth is also accompanied by temporal oscillations. At small wavenumbers the perturbations grow monotonically in time but soon saturate. Our results also apply to expanding relativistic jets as long as the opening angle of the jet is larger than the inverse of its Lorentz factor.

In the collapsar model of GRBs, a collimated relativistic outflow is generated by the collapse of the core of a massive star. The outflow approaches the stellar envelope at a modest semirelativistic speed but is expected to accelerate significantly across the sharp density gradient at the surface of the star (Tan et al. 2001; Zhang, Woosley, \& MacFadyen 2003 and references therein). Our results indicate that in the breakout phase, perturbations are close to being stable in spherical symmetry. It is still possible, however, that the lateral expansion of the jet at breakout would be accompanied by instabilities. These instabilities may produce variations in the Lorentz factor of the jet needed in the internal shock model. They may also be responsible for the complex light curves observed in most GRBs. Current numerical simulations (Zhang et al. 2003 and references therein) lack adequate resolution at the stellar surface to follow the shock breakout and confirm the instabilities. We leave a detailed study of the instabilities associated with the lateral expansion of the jet for future work.

This work was supported in part by grants from the Israel-US BSF (BSF 98-00343) and NSF (AST 00-71019, AST 02-04514).
Best, P., \& Sari, R. 2000, Phys. Fluids, 12, 3029

Blandford, R. D., \& McKee, C. F. 1976, Phys. Fluids, 19, 1130 (BMK)

Cox, J. P. 1980, Theory of Stellar Pulsation (Princeton: Princeton Univ. Press)

Johnson, M. H., \& McKee, C. F. 1971, Phys. Rev. D, 3, 858

Perna, R., \& Vietri, M. 2002, ApJ, 569, L47

Sari, R., Waxman, E., \& Shvarts, D. 2000, ApJS, 127, 475

Sedov, L. I. 1946, Prikl. Mat. Mekh., 10, 241

\section{REFERENCES}

Tan, J. C., Matzner, C. D., \& McKee, C. F. 2001, ApJ, 551, 946

Taylor, G. I. 1950, Proc. R. Soc. London A, 201, 159

Von Neumann, J. 1947, Los Alamos Sci. Lab. Tech. Ser., 7

Waxman, E., \& Shvarts, D. 1993, Phys. Fluids A, 5, 1035

Zeldovich, Ya. B., \& Raizer, Yu. P. 2002, Physics of Shock Waves and High-Temperature Hydrodynamic Phenomena (Mineola, NY: Dover)

Zhang, W., Woosley, S. E., \& MacFadyen, A. I. 2003, ApJ, 586, 356 